\title{
Diseño y caracterización de sistema de detección heterodino para interferometría de baja coherencia
}

\section{Heterodyne detection system characterization for low coherence interferometry}

Presentación: 6-7/10/2020

\section{Doctorando:}

\section{Leslie Judith Cusato}

Grupo de Fotónica Aplicada, Facultad Regional Delta, Universidad Tecnológica Nacional - Argentina Icusato@frd.utn.edu.ar

\section{Director/a:}

\section{Eneas Nicolás Morel}

\section{Co-director/a:}

\section{Jorge Román Torga}

\section{Resumen}

La interferometría de baja coherencia está generando considerable interés en aplicaciones industriales donde es necesario realizar medidas más grandes con mayor resolución. Los sistemas convencionales en el dominio de Fourier alcanzan una profundidad limitante de alrededor de $3 \mathrm{~mm}$, debido principalmente a los espectrómetros utilizados para la detección. En este trabajo se presentan los avances relacionados al diseño, desarrollo y caracterización de un sistema de detección heterodino el cual permite aumentar en un orden de magnitud el rango de trabajo.

Palabras clave: Interferometría. Tomografía de coherencia óptica. Detección heterodina.

\begin{abstract}
Low coherence interferometry is generating considerable interest in industrial applications where larger measurements with high resolution are needed. Conventional systems in the Fourier domain reach a limiting depth of $3 \mathrm{~mm}$, mainly due to the spectrometers used in detection. In this work, the advances related to the design, development and characterization of a heterodyne detection system are presented, which allows to increased the dynamic range in an order of magnitude.
\end{abstract}

Keywords: Interferometry. Optical coherence tomography, Heterodyne detection

\section{Introducción}

La interferometría de baja coherencia (low-coherence interferometry, LCI) es una técnica de medición óptica no destructiva capaz de alcanzar resoluciones micrométricas en la dirección axial. El objetivo principal de esta técnica es medir diferencias de 
camino óptico (optical path difference, OPD) para hallar distancias de interés en una muestra. Dada su naturaleza no invasiva, una de las aplicaciones principales es en el campo de las imágenes biomédicas, a través de la tomografía óptica coherente (optical coherence tomography, OCT) (Drexler \& Fujimoto, 2015). Sin embargo, esta técnica presenta diversas ventajas en una gran variedad de áreas como arte (Rouba et al., 2008; Targowski \& Wojtkowski, 2006), caracterización de componentes, perfilometría (Cerrotta et al., 2015; Nandi et al., 2009) y sensores de fibra óptica (Volkov et al., 2013)

Existen tres tipos de sistemas usados generalmente en LCI: en el dominio del tiempo (TD-LCI), en el dominio de Fourier (FDLCI) y con fuentes de barrido (SS-LCI). En el dominio del tiempo, la señal de interferencia es generada moviendo un espejo de referencia en uno de los brazos del interferómetro. El desarrollo de FD-LCI permitió el diseño de sistemas más robustos y compactos, sin partes móviles en la rama de referencia. En estos sistemas, se detecta mediante un espectrómetro el interferograma y se realiza la transformada de Fourier del mismo para hallar las OPD y en consiguiente las distancias de interés. En el caso de SS-LCI, la fuente de luz es sintonizable y se utiliza un único detector lo cual permite aumentar la velocidad de escaneo del sistema.

El rango de medición de esta técnica varía según el sistema utilizado. En TD-LCI para medir distancias mayores a un centímetro se necesitan sistemas de desplazamiento de gran precisión, pero extremadamente lentos. Esta limitación es superada usando sistemas FD-LCI. Sin embargo, el estado del arte de estos sistemas se encuentra en distancias del orden de $3 \mathrm{~mm}-4 \mathrm{~mm}$, principalmente a causa del espectrómetro utilizado en la detección: tanto la red de difracción como el número de píxeles afectan el muestreo de la señal. Otra gran limitación de esta técnica es el efecto "fall-off" debido al tamaño finito de los píxeles que provocan la atenuación de la señal para OPD largas.

Es de interés para muchas industrias, extender el rango de trabajo de esta técnica. Si bien es posible realizar esto utilizando SS-LCI, el costo del sistema se incrementa considerablemente. Estudios recientes han logrado duplicar el rango de trabajo de la técnica FD-LCI generando la señal compleja completa (Bachmann et al., 2006) o usando bandas de Talbot (Bradu \& Podoleanu, 2011). No obstante, estos métodos aún presentan las limitaciones impuestas por los espectrómetros usados en la detección.

En este trabajo, se presenta el estudio de un novedoso sistema de detección para la técnica FD-LCI basado en un espectrómetro espacial heterodino (Harlander et al., 1992). Este dispositivo realiza la transformada de Fourier de la señal, permitiendo obtener las distancias de interés sin necesidad de un post procesamiento. La principal ventaja de este sistema reside en la independencia del sensor utilizado, evitando así el efecto "fall-off" de los sistemas convencionales. Como resultado, el rango dinámico es incrementado en un orden de magnitud.

\section{Desarrollo}

FD-LCI es una técnica interferométrica que se basa en una fuente de luz de baja coherencia para medir OPD. Usualmente se utiliza un interferómetro de Michelson como muestra la figura 1(a). Las reflexiones producidas en la rama de referencia y en la muestra se recombinan después de pasar por el divisor de haz, generando la señal de interferencia detectada por un espectrómetro. Al realizar la transformada de Fourier del interferograma, se obtienen las OPDs características como muestra la figura 1(c).

En este trabajo se desarrolló un sistema de detección basado en un espectrómetro espacial heterodino. El mismo consiste en un arreglo interferométrico con redes de difracción como muestra la figura 1(b). 


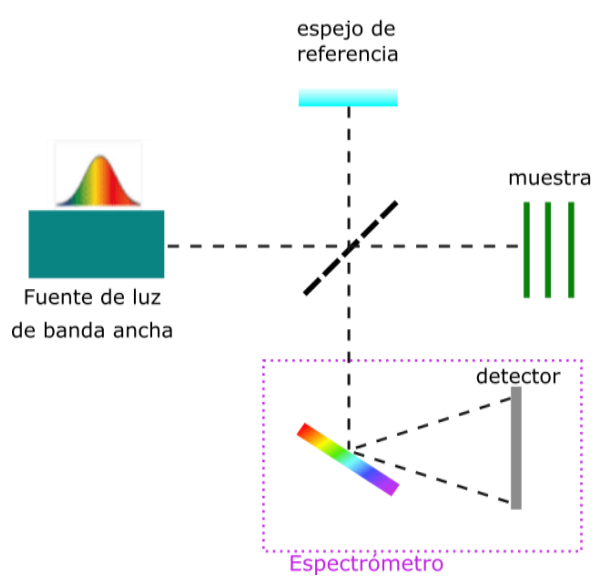

(a)

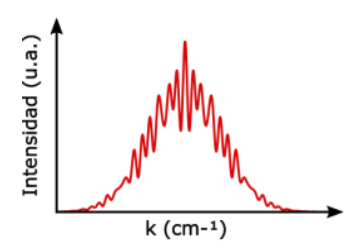

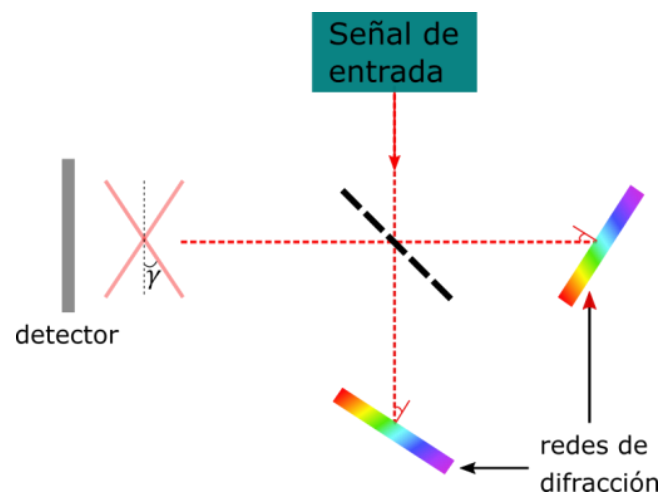

(b)

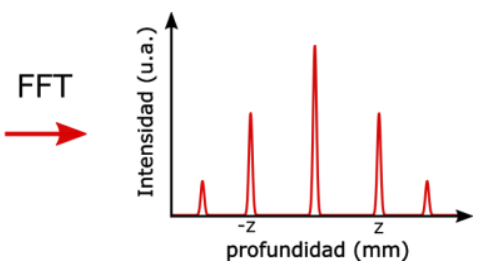

(c)

Figura 1: (a) Esquema experimental del sistema LCI convencional. (b) Dispositivo experimental del sistema heterodino de detección. (c) Representación de la señal interferométrica típica y las señales de interés obtenidas al realizar la transformada de Fourier.

Para estudiar el sistema, se desarrollaron simulaciones en el lenguaje de programación Python, que permitieron estudiar la incidencia de distintos parámetros sobre las señales a la salida. El modelo utilizado comprende dos secciones principales: una correspondiente al interferómetro donde se genera la señal y la otra al sistema heterodino de detección. La primera permite modelar la señal generada de entrada al sistema de detección teniendo en cuenta el espectro de la fuente de luz utilizada y la OPD de interés. Además, es posible modelar la presencia de elementos dispersivos con distintos índices de refracción en las ramas del interferómetro. La segunda parte del modelo permite modificar el diseño del sistema heterodino, variando la cantidad de líneas de las redes de difracción, su ángulo de inclinación y la distancia respecto al divisor de haz. Permite modificar también, el tamaño del haz que ilumina las redes y el tamaño del sensor utilizado.

El sistema de detección heterodino se montó junto al sistema LCI y se estudió su rango de trabajo utilizando un espejo en la rama de muestra. El mismo fue desplazado para generar distintas OPDs y emular distintas distancias de interés de una muestra. Para automatizar el proceso de medición, se desarrollaron rutinas en el lenguaje de programación Python que permitieron controlar el movimiento del espejo y las redes y sincronizarlo con la adquisición de imágenes de la cámara. Para analizar las señales adquiridas y hallar las distancias de interés, se estudiaron diversos métodos de procesamiento de imágenes. Una de las técnicas utilizadas se describe con detalle en (Cusato et al., 2019).

\section{Resultados}

El estudio del sistema mediante las simulaciones permitió conocer en profundidad las capacidades de este para optimizar su diseño. En la figura 2(a) se muestra la señal interferométrica simulada para una OPD de $0.2 \mathrm{~cm}$ al utilizar una fuente de ancho de 
banda $50 \mathrm{~nm}$ junto a la señal detectada por el sistema heterodino (b). Se observan dos términos espejo a cada lado del máximo central, correspondientes a la OPD del sistema LCI.
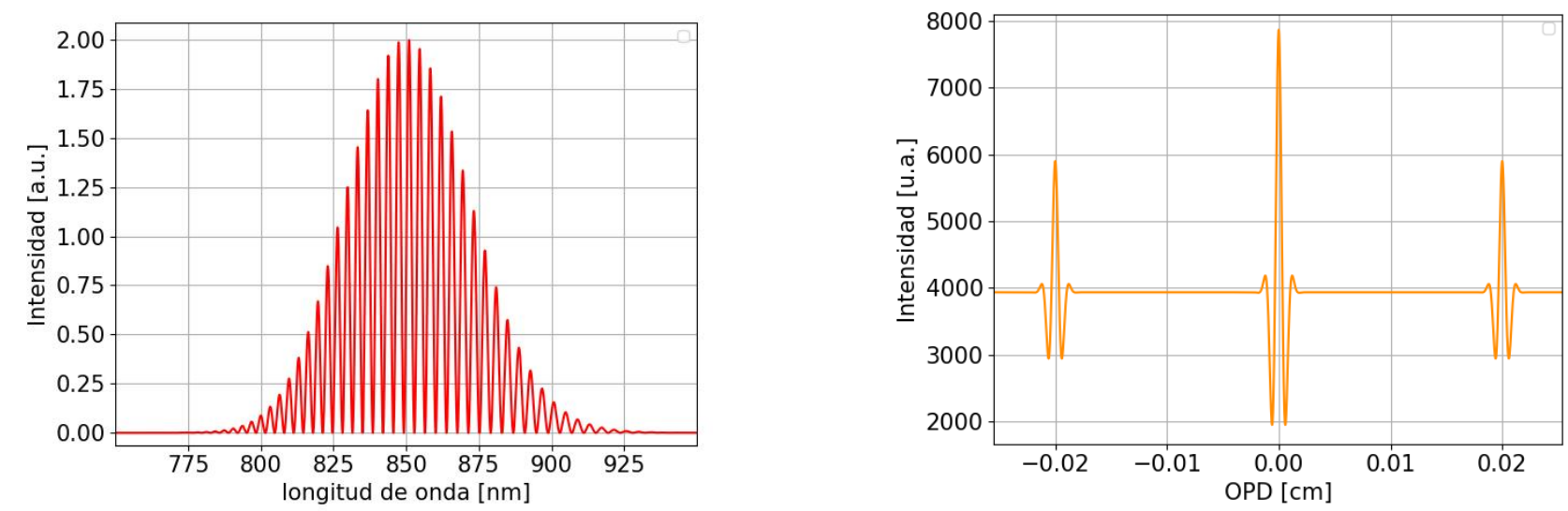

Figura 2: (a) Interferograma simulado para una OPD de $0.02 \mathrm{~cm}$. (b) Señal a la salida del sistema de detección heterodino. Se aprecian dos picos correspondientes a la OPD a ambos lados del máximo central.

Dado que el sensor utilizado fue una cámara, se simularon las imágenes a obtener en dos dimensiones (figura 3 a y b). Las oscilaciones internas de cada franja se deben a una variación en la inclinación de las redes en el eje perpendicular al eje óptico. En el caso en que no existe inclinación entre las redes, se espera obtener franjas verticales. En la figura 3 se compara las imágenes simuladas con las obtenidas experimentalmente (c).

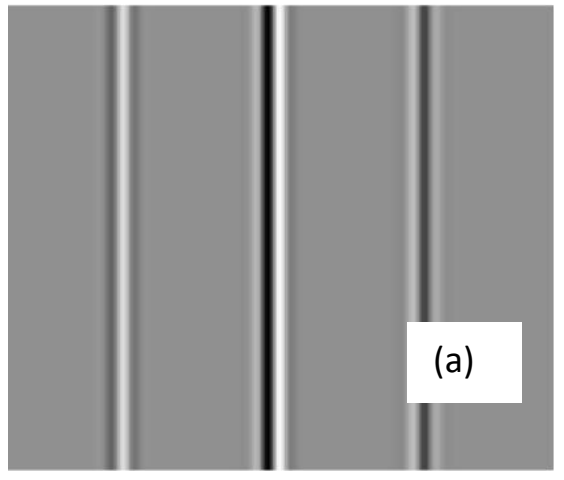

(a)

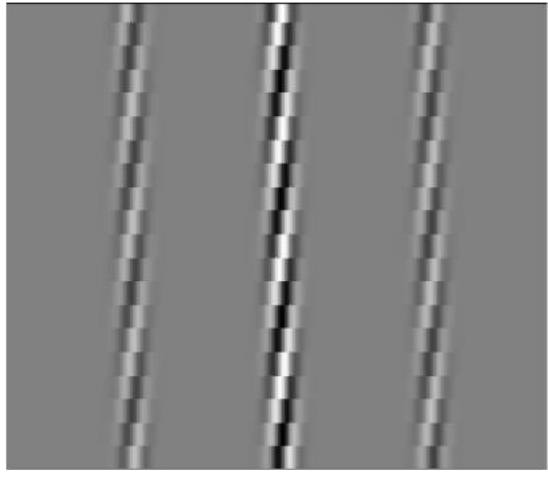

(b)

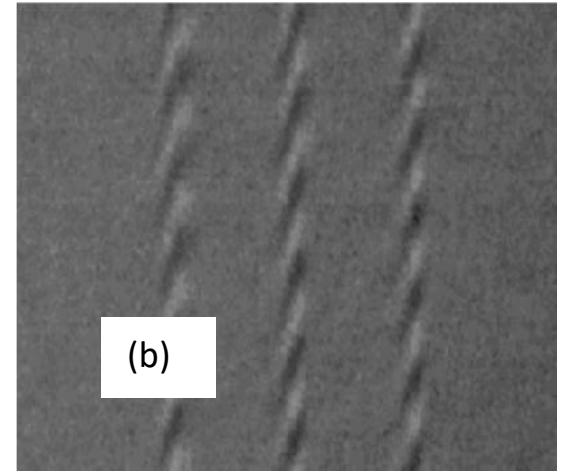

(c)

Figura 3: Comparación entre las imágenes de la señal detectada por el heterodino cuando las redes se encuentran sin (a) y con (b) inclinación en el eje vertical. En (c) se muestra la imagen adquirida experimentalmente, que concuerda con lo predicho.

Como parte del diseño del sistema de detección fue importante analizar qué incidencia tenía la posición de las redes sobre la señal adquirida. En la figura 4 se muestra la señal esperada para dos distancias distintas entre las redes y el divisor de haz. Se aprecia que, al ubicar la red más alejada del divisor, la señal pierde contraste y aumenta su ancho aparente. Esto se debe al espectro efectivo que finalmente es analizado por el detector heterodino. A medida que se agranda la distancia entre la red y el divisor de haz, las longitudes de onda son dispersadas en un área mayor y no logran incidir sobre el divisor de haz. Esto produce una reducción en el ancho de banda del espectro de entrada (Figura 4) que se traduce en un ensanchamiento en la señal. 


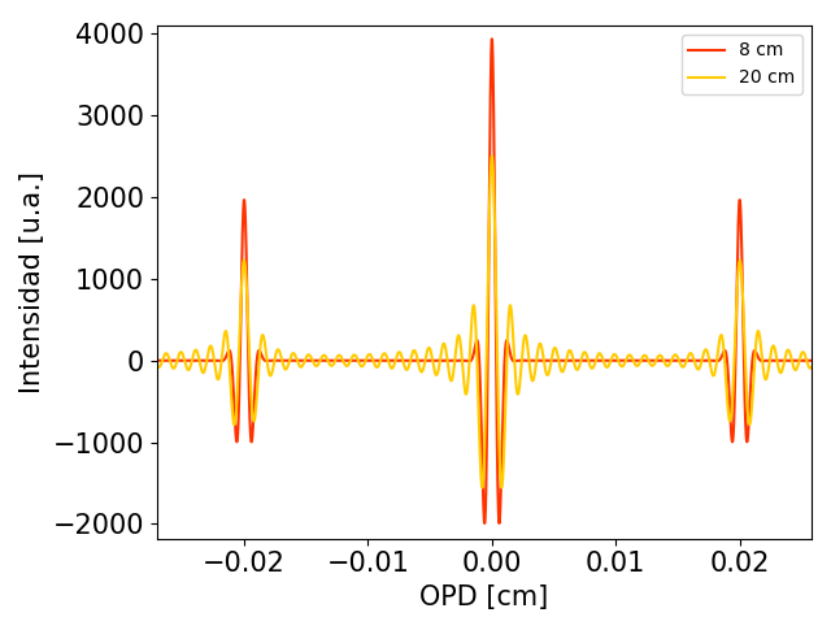

(a)

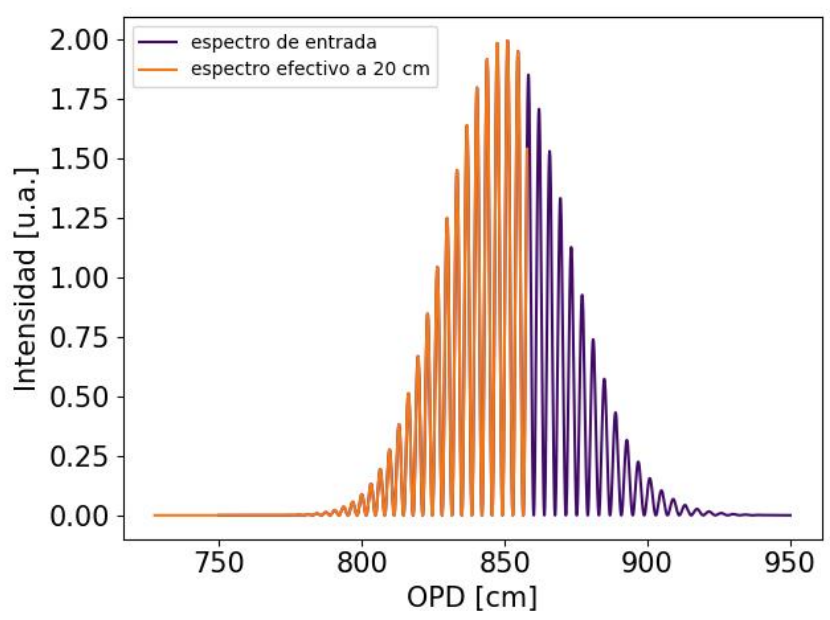

(b)

Figura 4: (a) Señal de detección para distintas distancias entre las redes de difracción y el divisor de haz. (b) Espectro efectivo comparado para la distancia más lejana. Se observa que se pierde parte del interferograma de entrada por las longitudes de onda que no logra atravesar el sistema.

Otro aspecto analizado fueron las variaciones del índice de refracción dentro del interferómetro LCI. En la figura 5 se muestran los resultados de las simulaciones al ubicar un tramo dispersivo de longitud $\mathrm{d}_{\mathrm{disp}}=2 \mathrm{~mm}$ con distintos índices de refracción (correspondientes a vidrio BK7 y NSF8) en el camino óptico hacia el espejo de muestra. Se observa que la posición del pico de interés se encuentra corrido respecto a la OPD esperada en el caso de medio no dispersivo $\left(\mathrm{n}_{\text {aire }}\right)$. Además, se aprecian cambios en el ancho de la envolvente de la señal de interés y en la frecuencia de la oscilación que la conforma.

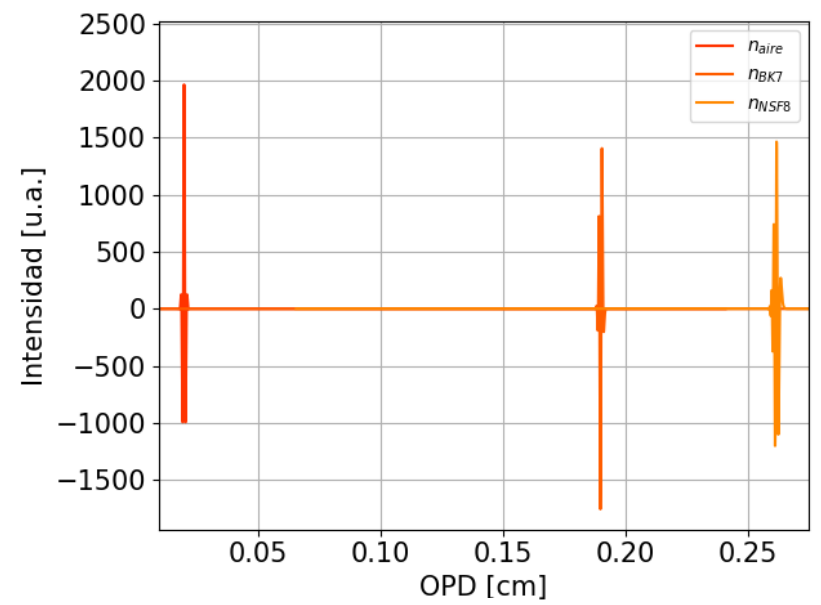

(a)

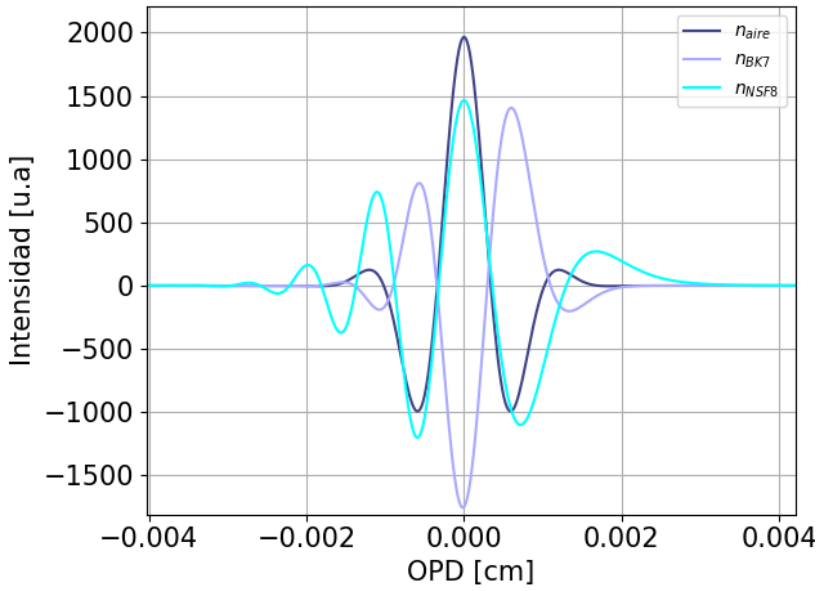

(b)

Figura 5: (a) Simulación de la señal adquirida para una misma OPD con un tramo dispersivo de $2 \mathrm{~mm}$ con distintos índices de refracción. (b) Comparación entre las señales donde se aprecia el cambio de ancho de le envolvente y la frecuencia de las oscilaciones que la conforman.

Para evaluar el rango de trabajo del sistema de detección heterodino se desplazó el espejo de la rama de muestra, cambiando así la OPD. La distancia máxima alcanzada por este sistema fue cercana a los $5 \mathrm{~cm}$ sin pérdida de resolución, un orden de magnitud 
mayor a los sistemas convencionales (Cusato et al., 2020) que alcanzan los $3 \mathrm{~mm}$ o $4 \mathrm{~mm}$. Esto se debe principalmente a la ausencia del efecto "fall off" debido al espectrómetro, presentando además ventajas con respecto a la sensibilidad.

\section{Conclusiones}

Se diseñó y desarrolló un sistema de detección heterodino para sistemas LCI que permitió aumentar en más de un orden de magnitud el rango de trabajo comparado con los sistemas convencionales.

El estudio del sistema mediante las simulaciones permitió conocer en profundidad las capacidades del mismo para optimizar su diseño. Se continuará trabajando sobre el análisis de las señales en medios dispersivos para estudiar la posibilidad de extraer información de interés sobre la muestra a través de las mismas.

\section{Referencias}

Bachmann, A., Leitgeb, R., \& Lasser, T. (2006). Heterodyne Fourier domain optical coherence tomography for full range probing with high axial resolution. Optics Express, 14(4), 1487. https://doi.org/10.1364/oe.14.001487

Bradu, A., \& Podoleanu, A. G. (2011). Attenuation of mirror image and enhancement of the signal-to-noise ratio in a Talbot bands optical coherence tomography system. Journal of Biomedical Optics, 16(7), 076010. https://doi.org/10.1117/1.3598446

Cerrotta, S., Morel, E. N., \& Torga, J. R. (2015). Scanning Optical Coherence Tomography Applied to the Characterization of Surfaces and Coatings. Procedia Materials Science, 9, 142-149. https://doi.org/10.1016/j.mspro.2015.04.018

Cusato, Leslie J., Morel, E. N., \& Torga, J. R. (2019). Preliminary study of a heterodyne detection system for optical coherence tomography signals. 2019 18th Workshop on Information Processing and Control, RPIC 2019, 193-197. https://doi.org/10.1109/RPIC.2019.8882138

Cusato, Leslie Judith, Cerrotta, S., Torga, J. R., \& Morel, E. N. (2020). Extending low-coherence interferometry dynamic range using heterodyne detection. Optics and Lasers in Engineering, 131(March), 106106. https://doi.org/10.1016/j.optlaseng.2020.106106

Drexler, W., \& Fujimoto, J. G. (2015). Optical coherence tomography: Technology and applications, second edition. In Optical Coherence Tomography: Technology and Applications, Second Edition. Springer International Publishing. https://doi.org/10.1007/978-3-319-06419-2

Harlander, J. M., Reynolds, R. J., \& Roesler, F. L. (1992). Spatial heterodyne spectroscopy for the exploration of diffuse interstellar emission lines at far-ultraviolet wavelengths. Astrophysical Journal, 396(2), 730-740.

Nandi, P., Chen, Z., Witkowska, A., Wadsworth, W. J., Birks, T. A., \& Knight, J. C. (2009). Characterization of a photonic crystal fiber mode converter using low coherence interferometry. Optics Letters, 34(7), 1123. https://doi.org/10.1364/ol.34.001123

Rouba, B. J., Karaszkiewicz, P., Tymińska-Widmer, L., Iwanicka, M., Góra, M., Kwiatkowska, E., \& Targowski, P. (2008). Optical Coherence Tomography for Non-Destructive Investigations of Structure of Objects of Art. 9th International Conference on NDT of Art.

Targowski, P., \& Wojtkowski, M. (2006). Optical Coherence Tomography for Artwork Diagnostics. Laser Chemistry, $2006,1-11$. https://doi.org/10.1155/2006/35373

Volkov, P. V., Goryunov, A. V., Luk'Yanov, A. Y., Tertyshnik, A. D., Baidakova, N. A., \& Luk'Yanov, I. A. (2013). Fiber-optic temperature sensor based on low-coherence interferometry without scanning. Optik, 124(15), 1982-1985.

https://doi.org/10.1016/j.ijleo.2012.06.043 